\title{
Technology-enhanced Support for Learning Conceptual Modeling
}

\author{
Gayane Sedrakyan, Monique Snoeck \\ Katholieke Universiteit Leuven, \\ Management Information Systems, \\ Naamsestraat 69, 3000 Leuven \\ E-mail: \{gayane.sedrakyan, monique.snoeck\} @econ.kuleuven.be
}

\begin{abstract}
This paper describes an optimized didactic environment to support and improve learning achievements for conceptual modeling. In particular, it describes computer-aided techniques to address various learning challenges observed in the teaching process such as: hybrid background of students, enrollment of a large number of students, the complexity of industrial tools and difficulties in abstract thinking. The didactic environment has been developed and subsequently optimized in the context of the course Architecture and Modeling of Management Information Systems. It includes 1) diagnostic testing with automated feedback 2) an adapted modeling tool 3) an MDA based simulation feature. The didactic tools were evaluated positively by the students and a positive impact was observed on the student's capabilities to construct objectoriented conceptual models.
\end{abstract}

Keywords. teaching business domain modeling, conceptual model, enterprise modeling, computer aided modeling, modeling tool, automated consistency control, managing knowledge diversity, automated feedback, model driven architecture, simulation, prototyping, executable models.

\section{Educational Context}

The main goal of the course "Architecture and Modeling of Management Information Systems"1 is to familiarize the students with modern methods and techniques of Object-Oriented Analysis and Design for Business Information Systems, to let them understand the relation between an information system and the organisational aspects of an enterprise, and to let them acquire sufficient skills of developing an

1 The course's page can be found on

http://onderwijsaanbod.kuleuven.be/syllabi/e/D0I71AE.htm 
enterprise model as basis of a business information system. This paper describes a didactic environment developed and subsequently optimized based on our experiences from observations of student achievements in the course over a period of 5 years, similar issues found in related research, and constant feedback from 300 students overall. It proposes an extension to the techniques previously presented by Snoeck et al. [1]. The methodology used is based on the concepts of Merode ${ }^{2}$.

The remainder of the paper is structured as follows. The second section presents the challenges observed in a learning process. Section 3 gives an overview of the related work and subsequently formulates our research question. Sections 4 to 6 describe optimization techniques for addressing the observed challenges. Section 4 describes a diagnostic testing environment allowing to detect various gaps in students' prerequisite knowledge in a self-regulating manner. Section 5 defines the goals of the adapted modeling tool and gives the overview of modeling techniques that support these goals as far as the modeling process and associated difficulties are concerned. Section 6 describes the prototyping feature that allows for model simulation and concludes with explaining how the tool improves the learning cycle through automated feedbacks and increased transparency between a model and its prototype. Section 7 then reports on our experiences and the evaluation of the didactic tools by the students. Finally, section 8 suggests some future directions.

\section{Problem Domain}

In view of the high demand for skilled IT professionals with skills in Enterprise Architecture, the faculty of Business and Economics decided to offer a one year program conducting a non-technical training in information management, with an emphasis on the efficient and effective application and management of information technology in various business contexts. The program admits students with an academic bachelor degree of any field and has been very successful in attracting students. As a result of the high number of students with diverse backgrounds, several challenges were observed hindering the realization of the course objectives which was previously taught to a small homogenous group of students with a bachelor in Information Systems. The major challenges observed include: A) hybrid backgrounds of students resulting in various gaps in prerequisite knowledge, subsequently leading to different levels of motivation; B) enrollment of a large number of students making it timeconsuming to provide frequent personalized feedback; up to more conceptual issues that include C) the complexity of industry modeling tools making these less effective in supporting a teaching process; D) theoretical knowledge being at high level of abstraction which makes it difficult to bridge the gap between abstract model and a concrete Information System, especially for the students who have never programmed before and thus lack in technical insight. In this paper we focus on computer-aided techniques to remediate the major difficulties of a learning cycle while also striving to

2 Merode is an Object Oriented Enterprise Modeling method. Its name is the abbreviation of Model driven, Existence dependency Relation, Object oriented DEvelopment. Cfr. http://merode.econ.kuleuven.be 
improve satisfactory learning outcomes. Our approach includes an optimized didactic environment that is well adapted to learning goals and is intelligent enough to guide throughout a modeling process. More specifically, the rest of this paper aims to describe how the learning cycle of a novice up to medium experienced learner can benefit from the combination of adaptation, automation and simulation techniques in the context of addressing the targeted learning challenges.

\section{Related work}

Several authors have pointed out the difficulties that come along with teaching a heterogeneous group of students in the context of analysis and design of business information systems [2-6] indicating that not handling substantial differences in prior knowledge between students will result in large differences in motivation and learning outcomes. To address this [8] proposes adapting teaching according to cumulative data on students' understanding and difficulties, while also highlighting the advantage of exploiting peer knowledge in addressing the diversity issue. Many researchers and practitioners also point to problems related to the UML [2-4] which has become a widely accepted standard for modeling. According to the complexity metric presented by [4] due to human cognitive load limitations the diversity of constructs and diagrams of the UML score from 2 to 11 times more complex than those of other methods. Furthermore, [9] points out that most of the modeling languages are too "noisy" with various concepts, that can result in misusing concepts and creation of unintended models, i.e. models that use the language concepts in a way not intended for the modeling domain. As proposed by the constructivist approach [7] the method of dialogue is the optimal way to address learning difficulties by delivering personalized feedback. However, as it can be concluded from the above-mentioned, teaching with industry UML tools would make it time-consuming in terms of addressing how-to-use questions, thus creating a risk to potentially mask the real objectives of the course. Our approach proposes automation technique to deliver feedbacks throughout the learning cycle. The use of a technology-enabled dialogue can improve the learning process in two ways. Firstly, the didactic tools can perform some routine tasks which were previously done by the instructor, leaving the instructor more time for in-depth discussions with the students (cfr. challenges A, B, C). Secondly and more importantly, the calculating power of a computer enables feedback and testing possibilities a human instructor cannot provide for (cfr. challenge B). Among the other fundamental deficiencies of UML is that it is unclear how to combine interactive, structural and behavioral aspects together in a single view [10]. As observed, this can lead to difficulties in mastering the behavioral aspect of modeling (cfr. challenge D). One of the effective approaches for improving the learning process of dynamic aspect includes the use of simulation technique. Benefits of simulation in optimizing the feedback cycle for learning modeling are demonstrated by [16]. To apply the technique we use the model-driven approach. Besides being able to transform a model into its executable form within the shortest development cycle which is essential in the context of limited timeframe of the course, model driven development also allows to enhance the 
understanding of the rationale behind system design decisions, as well as improve the correctness and completeness of a model [18]. We will therefore apply the adaptation technique to build a modeling environment that is 1) able to support model-driven transformation 2) can alleviate the problem of "noisiness" of UML by limiting to a restricted UML with a limited number of views 3 ) is intelligent enough to guide the student throughout modeling process.

Table 1. Coverage matrix of techniques used per challenge

\begin{tabular}{|c|c|c|c|c|}
\hline & $\begin{array}{l}\text { Diversity } \\
\text { (A) }\end{array}$ & $\begin{array}{c}\text { Large number of } \\
\text { students (B) }\end{array}$ & $\begin{array}{l}\text { Complexity of } \\
\text { UML tools (C) }\end{array}$ & $\begin{array}{l}\text { Abstract thinking } \\
\text { (D) }\end{array}$ \\
\hline $\begin{array}{c}\text { Diagnostic } \\
\text { Test }\end{array}$ & $\begin{array}{l}\text { automated } \\
\text { feedback }\end{array}$ & $\begin{array}{l}\text { automated feed- } \\
\text { back }\end{array}$ & $\begin{array}{l}\text { automated feed- } \\
\text { back }\end{array}$ & \\
\hline Modeling tool & adaptation & $\begin{array}{l}\text { automated feed- } \\
\text { back }\end{array}$ & adaptation & \\
\hline $\begin{array}{c}\text { Prototyping } \\
\text { tool }\end{array}$ & simulation & $\begin{array}{l}\text { automated feed- } \\
\text { back }\end{array}$ & simulation & $\begin{array}{l}\text { simulation / au- } \\
\text { tomated feedback }\end{array}$ \\
\hline
\end{tabular}

Finally, to operationalize these techniques, we propose a didactic environment that includes 1) automated diagnosing tests allowing to deal with prerequisite knowledge gaps in a self-regulating manner through automated feedbacks; 2) a modeling tool adapted to the learning goals with built-in intelligence; 3 ) a simulation feature providing increased transparency between a model and its dynamic behavior. Table 1 shows the coverage matrix of techniques used per challenge within the didactic environment.

\section{Diagnostic testing}

To achieve the objectives of the course students need to complete a set of modeling exercises. Exercises start with a textual description of some user requirements, which should be interpreted by the student and transformed into an object-oriented conceptual model. Given the fact that such user requirements can and will be interpreted in different ways (because of their informal aspect) by students, students should receive individual feedback on their solution. Unfortunately, both the number of students enrolled (cfr. challenge B) and the use of paper and pencil exercises hamper the realizations of these (course) objectives. Furthermore, students have very different levels of prior knowledge at the beginning of the course (cfr. challenge A). To address the problems of various gaps in students' prerequisite knowledge (cfr. challenge A) a self-regulating approach was stimulated among students to detect and recover possible gaps. This was achieved by developing a testing environment to motivate and help students to discover to which extent they lack prerequisite knowledge. A sample diagnostic test examining knowledge in ER modeling is shown in Fig. 1. Through the tests, students are guided by immediate automated feedbacks as well as tips for improvements. Examples of automated feedbacks to student are shown in Fig. 2. The 
method showed positive impact on the learning process in terms of stimulating a selfregulated manner towards prerequisite knowledge.

Answer the 'yes/no'-questions concerning the following UML class diagram to test your knowledge. (read the questions carefully before answering)

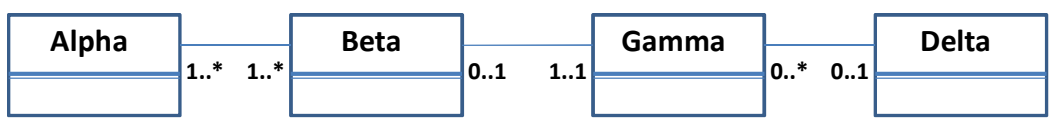

Fig. 1. Example of a diagnostic test

Students with deficient prerequisite were offered extra sessions at the start of the course. Subsequently, the smaller number of (the right) students attending these sessions facilitated the dialogue with the teacher. With the diagnostic environment, however, the number of participants increased, and multiple self-testing cycles were registered by the system for the same student indicating gradually improving results with each cycle.

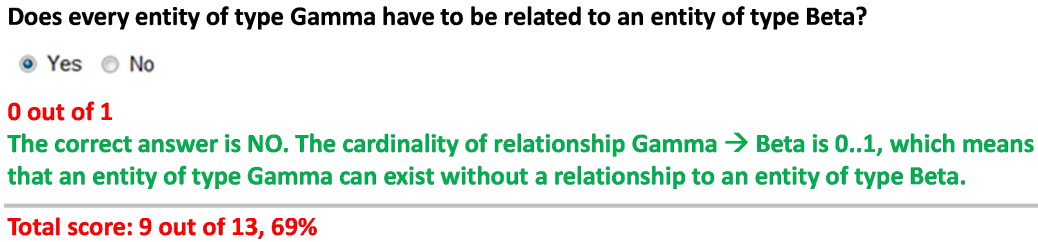

You should consider following the catch-up session on ER concepts on the 11th of October (9am), or revisioning Appendix A in "Object-oriented Enterprise Modelling with MERODE".

Fig. 2. Example of an automated feedback of a diagnostic test

Additionally, the diagnostic environment allows to store cumulative data about the students prerequisite knowledge. Having data about students understanding, skills and difficulties, in turn, helps to adapt teaching accordingly [8]. In addition to the diagnostic tests, throughout the semester peer dialogue was used as an alternative source for feedbacks in the context of group assignments by means of review sessions among the groups. Diversity in this context, as observed by several studies, can create an advantage for exploiting a "peer expertise". Furthermore, peer dialogues can affect the learning process in a variety of ways by 1) creating an opportunity for broadening student's mind with alternative perspectives and tactics, 2) communication being conducted on the "same language" that makes concepts easy to grasp and critique less stressful to accept, 3) enabling to revise or reject hypothesis, 4) developing detachment of judgment (about work in relation to standards) by commenting on the works of peers [8]. Furthermore, peer reviews for group solutions allow to teach students skills "to read models, not only to write them" [3]. In addition to the offline peer reviews, peer communication was also supported by enabling technologies, such as Wikis. 


\section{$5 \quad$ Adapted modeling tool}

Development of an own adapted modeling environment was motivated by the need for addressing the learning challenges related to the complexity of industry tools as well as the need to support an MDE-based prototyping feature allowing to visualize the dynamic aspect of a model (see section 6 on prototyping). Advantages of own environment over the industry tools include: 1) modeling techniques adapted to learning goals 2) restricted number of easily traceable views allowing to complete a modeling task through incremental and iterative steps, 3) automated feedbacks and built-in model checking mechanism that guides a student throughout a modeling process, 4) a prototyping feature allowing to visualize/validate a model based on its dynamic aspect (cfr. challenge D).

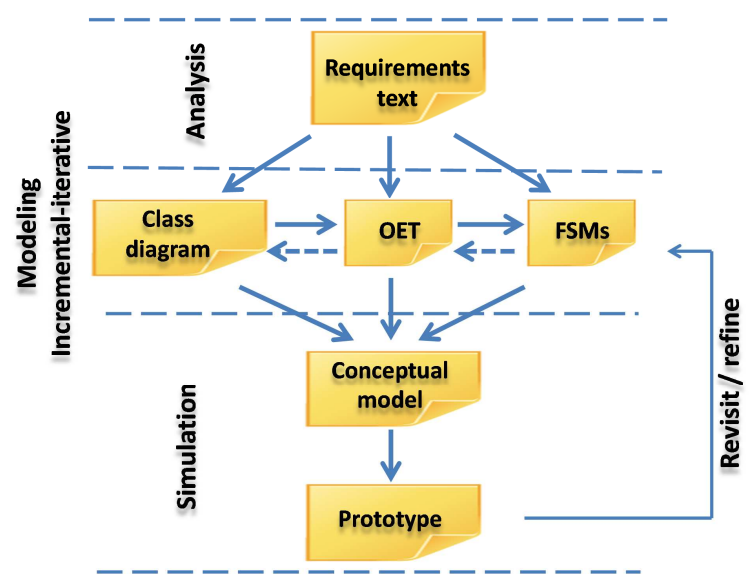

Fig. 3. Artefacts and modeling cycle with Merode

Fig. 4. depicts the artefacts and modeling cycle with Merode within the proposed adapted environment. An object-oriented business model typically consists of several views that together define a platform independent model that is a formal representation of the user requirements. In the modeling method used in this course, an enterprise domain model consists of a class diagram, an interaction model and a number of state charts. The class diagram is a restricted form of UML class diagram: the types of associations are limited to binary associations, with a cardinality of 1 to many or 1 to 1 . Many to many associations need to be converted to an intermediate class. The interaction model consists of an Object-Event Table (OET), created according to the principles of Merode [11]. It represents a kind of CRUD-matrix ${ }^{3}$, a technique borrowed from Information Engineering [12]. In Merode, "business events"

3 CRUD-matrix is a table containing functions of an application and with each function entry indicating the type of interaction with a database for that function: Create, Read, Update or Delete. 
represent atomic actions from the real world in which one or more domain objects can participate. Each business event is assigned an owner class indicated by an "O/" pre-

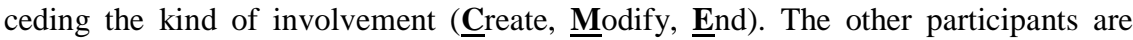
considered as "Associated" participants and have the C, M or E preceded by "A/".

\section{Class diagram}

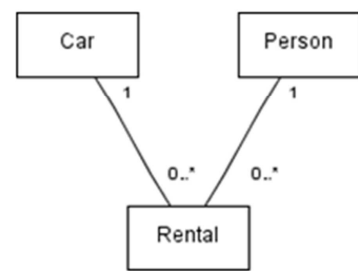

FSM

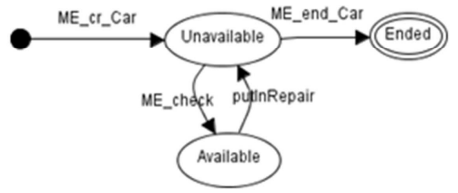

OET

\begin{tabular}{|c|c|c|c|}
\hline & 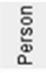 & ১ొ & 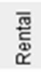 \\
\hline EV_cr_Person & O/C & & \\
\hline apply & $\mathrm{O} / \mathrm{M}$ & & \\
\hline update_info & $\mathrm{O} / \mathrm{M}$ & & \\
\hline approve & $\mathrm{O} / \mathrm{M}$ & & \\
\hline disapprove & $\mathrm{O} / \mathrm{M}$ & & \\
\hline EV_end_Person & O/E & & \\
\hline EV_cr_Car & & $\mathrm{O} / \mathrm{C}$ & \\
\hline check & & $\mathrm{O} / \mathrm{M}$ & \\
\hline put_In_Repair & & O/M & \\
\hline EV_end_Car & & O/E & \\
\hline EV_cr_Rental & AIM & AlM & o/C \\
\hline EV_end_Rental & AIM & AMM & O/E \\
\hline
\end{tabular}

Fig. 5. Examples of class diagram, Object-Event Table (OET) and a Finite State Machine (FSM) supported by JMermaid modeling tool

The finite state machines allow the object type to impose sequence constraints on the business events it is involved in. Multiple Finites State Machines (FSMs) allow to model independent aspects as parallel machines.

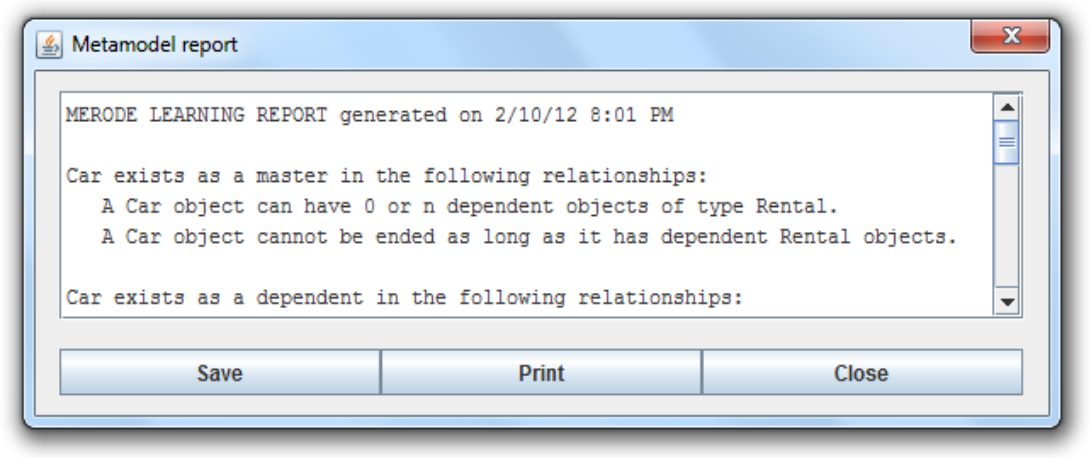

Fig. 6. Example of learning report

While modeling, student should receive interactive feedback on the quality of the developed model. The graphical editors of the adapted JMermaid ${ }^{4}$ tool have built-in

4 Cfr. http://merode.econ.kuleuven.be/mermaid.aspx 
intelligence that prevent from entering inconsistent specifications. In order to provide students with an elementary form of feedback, a model-to-text feature which converts a model to plain English has been provided. In previous years we experienced that "reading" the data view aloud ( like "This model says that... Is that what you meant?"), very often was sufficient to make students realize obvious mistakes. Especially students who are totally unfamiliar with modeling benefit from this feature. This model-to-text facility is called the "learning report" and has been implemented for the data view (see Fig. 6). Students can request for a learning report to pop up each time when drawing an association between classes, or can request a learning report for the data view as a whole.

A second feature concerns the tool's intelligence for managing consistency between the three views of the universe of discourse: the data view, the behavioral view and the interaction view. In its initial form, the tool followed a "consistencyby-construction" approach [13], [14]. In this approach, each time when entering specifications in one view, specifications that can be derived for other views are automatically generated by the tool. As an example, one of the design guidelines states that when defining a class, one should provide at least one method to create instances of that class and one method to terminate instances. Also the behavioral modeling with finite state machines has been augmented with a number of verification tools. The first set of tools act on a finite state machine and report about anomalies in the diagram: forward and backward inaccessible state, non-determinism and missing methods. Next, if multiple finite state machines are used to model parallel aspects of a single class, the student can request the tool to calculate the global behavior implied by the parallel composition of the individual finite state machines. Finally, the tool allows a student to run a check over the complete model to generate a tip-based report on the overall internal consistency of a model.

\section{Validation through simulation}

One of the major goals of the course is that students achieve the capability to mentally transform the abstract concepts into a concrete Information System (D). We delegated this to the method of simulation because of the recognized advantage of the latter to improve feedback cycles for learning the dynamic aspect of a model. The advantage of learning based on system dynamics is emphasized by many studies. In particular, among other benefits, simulation makes it possible to 1) trace from model to its effects in the concrete system, 2) conduct experiments and test the system for several alternatives with "what-if" scenarios, 3) increase model accuracy through detection of errors at early phases of engineering, and finally 4) it motivates the use of a simulator with the need to gain enough experience that usually cannot be gained by reading or lecturing alone [15], [16]. 


\subsection{MDA-based Prototyping with Merode}

Although many tools are able to generate fairly easily simple data-oriented prototypes no tool is capable of generating prototypes that are able to simulate both the data-aspects and the behavioral aspects contained in the finite state machines. To master the behavioral aspect of a domain model, the student needs to grasp how the overall behavior of a system results from the individual behavior of objects, all running in parallel while behaving according to the finite state machine associated with their class and synchronizing when jointly involved in business events. Since the behavioral aspects of the model is reported as the most difficult subject to master, being able to simulate also the behavioral aspects is of utmost importance. Hence, in order to assist the student in building a mental model of both the data and the behavioral view, the prototyping feature generates a Java application using a code generator based on the Model Driven Architecture (MDA) [17]. Transformation to prototype code can be achieved through a single click: the student receives a ZIP-archive, containing both a compiled application in executable JAR format and the source-code.

\subsection{Relationships between model and prototype}

Before we discuss the transformed behavior of model we give a short overview of the structure of a generated prototype application. It consists of three layers, a graphical user interface (GUI) layer, an event handling layer and a persistence layer.

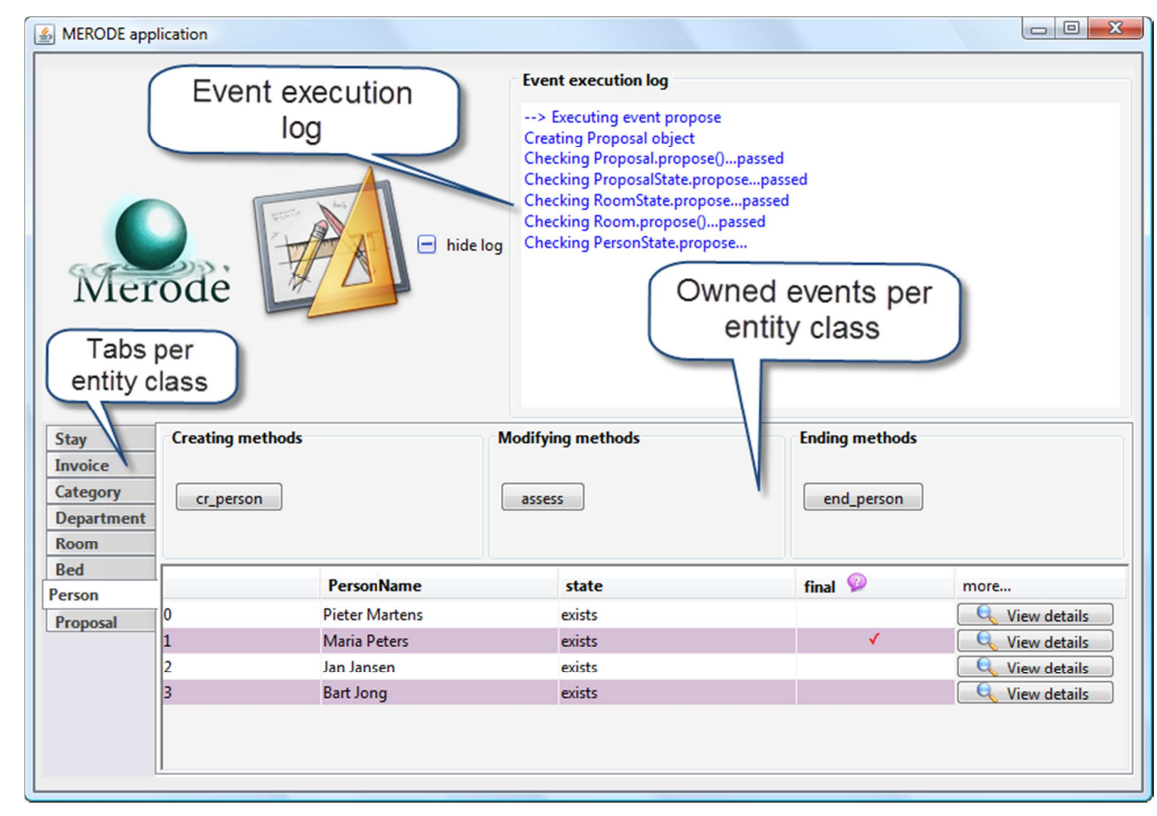

Fig. 7. Main window of a prototype application 
The graphical user interface has only basic functionality like triggering the creating and ending of objects, and triggering other business events. The GUI layer is built on top of the event handling layer. The task of the latter layer is to handle all events correctly by managing the appropriate interactions with the objects in the persistence layer. Student interacts with the generated application through the graphical user interface (GUI).

The event handling layer consists of a collection of so called event handlers. The working of an event handler can be described in four steps: 1) Upon an event execution call the event handler 'asks' every participating object (the participants to a business event that have been specified in the Object-Event Table) whether all preconditions set by the object are met. For example, associations between classes will lead to preconditions to maintain referential integrity; 2) Similarly to the previous step the event handler retrieves from every participating object its current state (or reference to the corresponding state object) and checks whether that state allows further processing of the event; 3) If all results of the tasks in step 1 and 2 are positive, the event handler invokes the methods in the participating objects, i.e. corresponding event triggered in response to processing the originally called event in the specific object; 4) next, if all results of previous steps are positive, the event handler executes the method in all participating objects retrieved in step 2 to implement the state modifications (according to the triggered event). While executing a business event in a prototype application users (or students) can follow in an event execution log frame what is happening in the upper right corner of the generated application.

\subsection{Increased transparency between model and prototype}

One of the methods of promoting better insight on the dynamic aspect of modeling was considered to be the increased transparency of the links between the model and its generated prototype application. During the course it was observed that students experience significant difficulties in linking the knowledge on validation rules gained from theory with the background process of validation in the prototype application. The connection proved to be not obvious with warning and error messages packaged with the generated prototype. Initially those violations were reported to students in the form of error popups (e.g. "Can't execute event: object in wrong state.") which were not easily interpreted by students, causing confusions up to the feeling that the prototype is not behaving as expected. This was partially because students also tend to draw links between the prototype application and its desirable behavior as would be expected in reality, at that level not being skillful enough to link to the errors coming from the model they designed themselves one reason being that during testing the prototype, the model was well hidden from the sight and mind. To make an analogy: a student walking in a (prototype) house discovering that there is no stair leading to the upper floor would simply conclude that the prototype was wrongly built, whereas (s)he should then check whether (s)he actually designed a stair on the plan for the house. Thus, while considering automated feedbacks for a prototype, the major emphasis was put on the failures caused by invalid calls of events. We believed that this would significantly improve the learning process, since the improved system of error 
reporting, in turn, would motivate to use the prototype application for better understanding, and subsequently for improving learning achievements with a purpose to detect defects. This will subsequently stimulate several improvement cycles of revisiting and refining a model while also allowing to gain a solid knowledge on dynamic behavioral aspect and mastering the necessary skills for modeling.

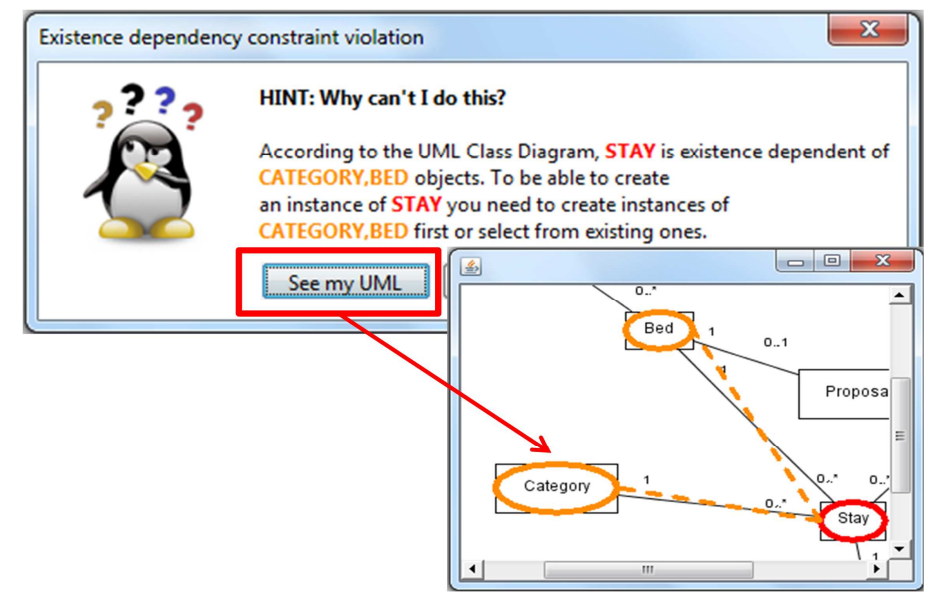

Fig. 8. Example of an automated feedback on 'mandatory one' rule violation

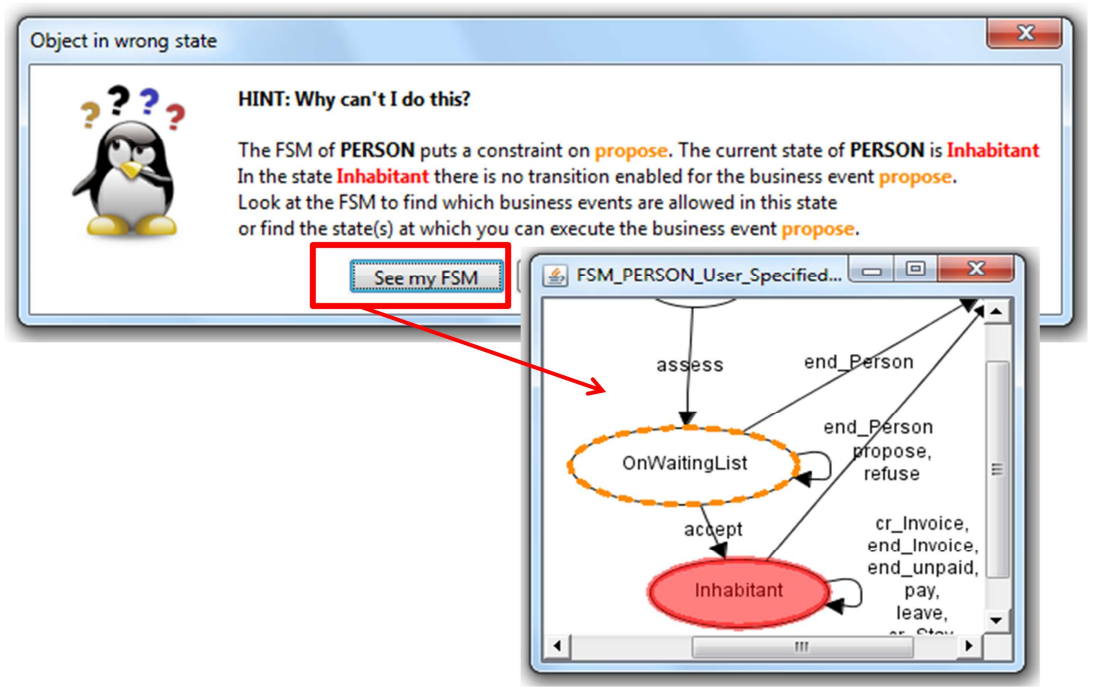

Fig. 9. Automated feedback on event execution being refused by object state 


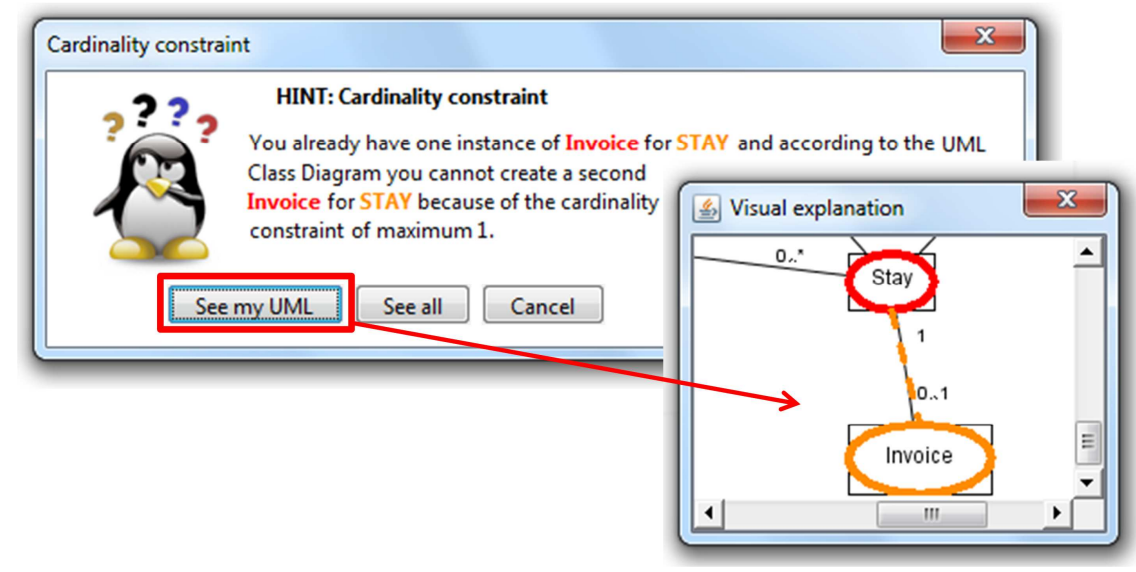

Fig. 10. Example of feedback on cardinality constraint violation

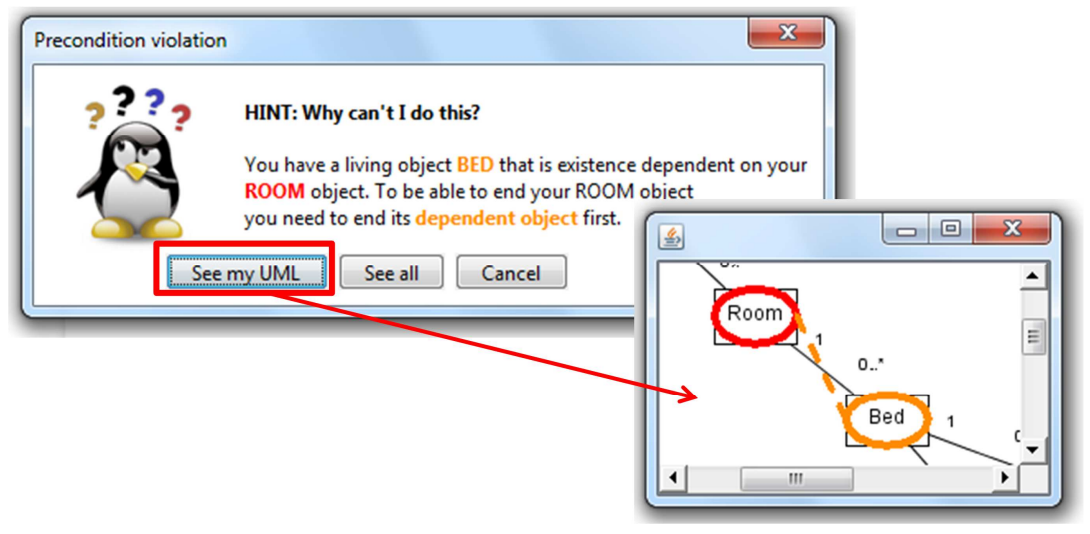

Fig. 11. Example of feedback on ending event failure due to living referring object

With the implementation of tip-based and visualized error reports students are gently guided into the causes of errors through: 1) more descriptive explanations in the error popups with an indication of the names of objects and/or events causing the problem; the explanations are reported in the coloring format linking to their visual representations 2) visual representation of the links between the errors and designed model 3) an image gallery that adds extra comfort by linking to the conceptual model and making it easy to navigate between a prototype and snapshot views of a model.

The set of implemented visualizations include feedbacks on failures that can result from: 1) Mandatory one cardinality violation: an object is attempted to be created before the objects it needs to refer to are created or associated (see Fig. 8). 2) wrong state of the claiming object: event execution fails because the state of the owner object or one of the associated participating objects refuse the execution of an event (see Fig. 9). 3) Cardinality violation: create-event execution fails due to a cardinality con- 
straint of maximum 1 (see Fig. 10). 4) Referential Integrity: ending-event execution fails due to existing referring objects. Example of automated report is shown on Fig. 11. The image gallery adds extra comfort by linking to all the views of the conceptual model, thus eliminating the need to switch between the application view and JMermaid modeling tool views.

\section{$7 \quad$ Evaluation and experiences}

The optimization of the didactic environment is based on constant feedbacks from students, experiments including proposed features and observations of a progress curve of delivered results from tasks and exams. The students were requested to evaluate each feature of the didactic tools on a range of 1 (not useful) to 5 (very useful). Table 2 shows the results of the evaluation for three years of teaching based on the answers of 92 participated students. The empty cells indicate that the feature was not available at the time of evaluation.

Table 2. Evaluation of didactic environment by students

\begin{tabular}{|l|c|c|c|}
\hline $\begin{array}{l}\text { How helpful were the following features ? } \\
(1=\text { Not O 5 = very helpful) }\end{array}$ & Year 1 & Year 2 & Year 3 \\
\hline Diagnostic Tests & - & - & 4,42 \\
\hline Peer Feedbacks & - & - & 3,29 \\
\hline Show learning dialog after inserting dependency & 3,6 & 3,6 & 3,94 \\
\hline Generate creating / ending events automatically & 4,6 & 4,6 & 4,18 \\
\hline Generate creating / ending methods automatically & 4,7 & 4,5 & 4.22 \\
\hline Create default finite state machines & 3,8 & 3,8 & 3,95 \\
\hline Generate model report & 3,7 & 3,3 & 3,7 \\
\hline Verify All: Consistency Report & 4,1 & 4,2 & 3,97 \\
\hline OET: Check methods tool & 4,4 & 4,5 & 3,54 \\
\hline FSM: Check FSM tool & 3,5 & 4,3 & 3,97 \\
\hline FSM: Calculated FSM tool & 2,6 & 4 & 3,39 \\
\hline $\begin{array}{l}\text { Is the use of JMermaid tool in general helpful in } \\
\text { learning the method? }\end{array}$ & 3,9 & 4,5 & 4,14 \\
\hline Code Generator / Rapid Prototyping & - & 3,7 & 3,46 \\
\hline Graphical visualizations of errors in prototype & - & - & 3,87 \\
\hline
\end{tabular}

With slight variations from year to year, all the features score largely above 3 (neutral), meaning that they are perceived as useful. The only concern was the popularity of the prototyping feature among the students. Previously the simulation was achieved through a chain of several transformation and execution steps before being able to run the prototype. The prototyping process was in addition complicated by an extra dependency of a generated prototype on an application server. Furthermore, the graph- 
ical visualizations of errors were implemented as an optional plugin students could extend their prototypes with. Due to their low technical skills, students experienced various difficulties throughout the simulation process chain, which made the major part of students reluctant in using the feature mostly resulting in "didn't use" answer while evaluating the feature. Despite these early problems the prototyping and errors' visualizations were rated above average (3,46 and 3,87 out of 5). Furthermore, a little experiment conducted with students before and after the use of simulated model resulted in the positive correction of 1,16 in the interpretation of a model, increasing from 7,63 to 8,59 in a range of $0-10$. In the meantime, the problems with the simulation chain have been solved by providing students with an all-in-one package allowing to generate and start a prototype with a single click from the student side. We therefore expect this tool to score even better in 2012 resulting in a much higher positive correction. Yet the growth in average grade on exams is another indicator of improved learning achievements along with significantly increased difficulty level of tasks that students are capable to handle.

\section{$8 \quad$ Future directions}

Although the applied techniques showed improvement in learning achievements, the didactic environment can be improved further and several issues still remain unaddressed. In the future we intend to further increase the intelligence of our didactic tools. Some possible directions include allowing increased interaction with a prototype generator to promote better technical insight. Taking into consideration the differences in prior knowledge the code generator can be extended to support different levels of technical expertise (cfr. challenge A) such as novice, intermediate, and advanced. This may be achieved by allowing a novice user to modify a simple GUI component or a popup message for the generated prototype, followed by intermediate level interaction such as a built-in Query Tool enabling manual queries to be sent to the prototype database, up to more advanced level management, such as (re)defining the structure of generated application, altering the database storage, modifying or adding templates in the template pool of the generator, e.g. a student may be allowed to add invariants and constraints through a template manager interface. We believe those can be good incentives motivating the students to model and experiment with the prototype while mastering skills that are not only related to the architecture and modeling aspects but also involve the domain of other courses taught in parallel, such as database systems, object-oriented programming, etc. thus stimulating the use of integrated knowledge which in turn will establish a more pragmatic and comprehensive focus among the students. Automation technique can be further extended to provide guidance throughout the requirements analysis phase. In parallel with experiments in an educational environment we consider validation and improvement of the proposed environment from the industry perspective. Yet another direction could be the integration of UML and JMermaid, enabling the latter to process an output of a standard UML tool. 


\section{References}

1. Snoeck, M., Haesen, R., Buelens, H., De Backer, M., Monsieur, G.: Computer Aided Modelling Exercises. Journal Informatics in education, Volume 6 Issue 1, 231 - 248 (2007)

2. Siau, K., Loo P.-P.: Identifying difficulties in learning UML. Information Systems Management 23 (3), 43-51 (2006)

3. Moisan, S., Rigault, J.-P.: Teaching Object-Oriented Modeling and UML to Various Audiences. Models in Software Engineering: Workshops and Symposia at MODELS, Denver, CO, USA (2009)

4. Erickson, J., Siau, K.: Can UML Be Simplified? Practitioner Use of UML in Separate Domains. In Proceedings EMMSAD'07, Trondheim, Norway, 87-96 (2007)

5. Wang, Sh.: Teaching the object-oriented approach for Business Information Systems Analysis and Design. Journal of Informatics Education and Research, Volume 4, N 1, IAIM Press (2002)

6. Secundo, G., Margherita, A., Passiante, G.: Hybrid Knowledge, Process and Technology: A Way to Managing Learners' Diversity. Research, Reflections and Innovations in Integrating ICT in Education, Published by FORMATEX, Badajoz, Spain (2009)

7. Hadjerrouit, S.: Object-Oriented Software Development Education: a Constructivist Framework, Informatics in Education, Vol. 4, No. 2, 167-192 167 (2005)

8. Nicol, D. J., Macfarlane-Dick, D.: Formative assessment and self-regulated learning: A model and seven principles of good feedback practice. Studies in Higher Education, Vol 31(2), 199-218 (2006)

9. Buckl, S., Matthes, F., Schweda, C. M.: A Meta-language for EA Information Modeling State-of-the-Art and Requirements Elicitation. In proceedings EMMSAD 2010, held at CAiSE 2010, volume 50 of Lecture Notes in Business Information Processing, 169-181, Springer (2010)

10. Gustas, R.: Conceptual Modeling and Integration of Static and Dynamic Aspects of Service Architectures. In Proceedings of ONTOSE, 17-32 (2010)

11. Snoeck, M., Dedene, G.: Existence dependency: the key to semantic integrity between structural and behavioural aspects of object types. IEEE Trans. Software Eng., 24(4):233-251, (1998)

12. Martin, J.: Strategic Data Planning Methods, Prentice-Hall, Inc., Englewood Cliffs, New Jersey, (1982)

13. Snoeck, M., Michiels, C., Dedene, G.: Consistency by construction: the case of MERODE, in Jeusfeld, M. A., Pastor, O., (Eds.) Conceptual Modeling for Novel Application Domains, ER 2003 Workshops ECOMO, IWCMQ, AOIS, and XSDM, Chicago, IL, USA, October 13, Proceedings, 2003 XVI, 410 p., Lecture Notes in Computer Science, Volume 2814, pp.105117 (2003)

14. Haesen, R., Snoeck, M.: Implementing Consistency Management Techniques for Conceptual Modeling, accepted for UML2004: 7th conference in the UML series, Lisbon, Portugal, October 10-15, (2004).

15. Merrill, D., Collofello, J.S.: Improving Software Project Management Skills Using a Software Project Simulator, Frontiers in Education Conference (1997)

16. Neu, H. Becker-Kornstaedt, U.: Learning and Understanding a Software Process through Simulation of Its Underlying Model. In Proceedings of LSO, 81-93 (2002)

17. OMG, Model-Driven Architecture, http://www.omg.org/mda/

18. Zikra I., Stirna J., Zdravkovic J.: Analyzing the Integration between Requirements and Models in Model Driven Development. In Proceedings BPMDS 2010, EMMSAD 2010, number 81 in Lecture Notes in Business Information Processing, 342-356, Springer (2011) 